\title{
Suresmile Technology: Precision to your Smile - A Review
}

\author{
Nor Syakirah Binti Shahroom ${ }^{1}$, L. Leelavathi ${ }^{2}$ and Saravanan Pandian ${ }^{3}$ \\ ${ }^{1}$ Saveetha Dental College and Hospitals, Saveetha Institute of Medical \\ and Technical Sciences, Saveetha University, Chennai 600077, India. \\ ${ }^{2}$ Senior Lecturer, Department of Public Health Dentistry, Saveetha Dental College and Hospitals, \\ Saveetha Institute of Medical and Technical Sciences, Saveetha University, Chennai 600077, India. \\ ${ }^{3}$ Senior Lecturer Department of Orthodontics, Saveetha Dental College and Hospitals, Saveetha \\ Institute of Medical and Technical Sciences, Saveetha University, Chennai 600077, India.
}

\section{ABSTRACT}

Dentistry has contributed to the huge advancement of technology and innovation for the past few decades. Suresmile technology is one of the latest advanced technologies in orthodontic treatment which has been introduced since 1998 and commercially released in 2005. The latest advancements in Dentistry other than Suresmile technology (OraMetrix) are Insignia (Ormco), orthoCAD (Cadent) and iBraces (3M Unitek) It uses advanced technology such as three-dimensional (3D) imaging of the dentition, robotics, and computer-aided manufacturing and digital model technology. It has been used widely by orthodontists and it may be expanded all over the world in the future. The advantages of this technology which provides benefits to the patients especially in terms of length of treatment make the patient willing to pay greater cost just for shorter treatment time. They might feel comfortable throughout the treatment as the number of visits to the dentist can also be reduced by using Suresmile. It is important to understand the accuracy and reliability of these imaging devices. Thus, proper knowledge about this technology is at utmost essential to ensure the great and successful outcome of the orthodontic treatment. Hence, a review was done to analyze the effectiveness, efficiency, reliability, advantages and disadvantages of the sure smile technology.

\section{KEY WORDS: CONVENTIONAL; ORASCANNER; ORAMETRIX; ORTHODONTICS; SURESMILE.}

\section{INTRODUCTION}

Dentistry has contributed to the advancement of technology and innovation for the past few decades. The latest advancements in Dentistry are Suresmile technology (OraMetrix), Insignia (Ormco), orthoCAD (Cadent) and iBraces (3M Unitek) (Sachdeva et al.,

\section{ARTICLE INFORMATION}

*Corresponding Author: leelavathi.sdc@saveetha.com Received 8th Aug 2020 Accepted after revision 26th Sep 2020 Print ISSN: 0974-6455 Online ISSN: 2321-4007 CODEN: BBRCBA

Thomson Reuters ISI Web of Science Clarivate Analytics USA and Crossref Indexed Journal

\section{Clarivate
Analytics}

NAAS Journal Score 2020 (4.31) SJIF: 2020 (7.728)

A Society of Science and Nature Publication,

Bhopal India 2020. All rights reserved.

Online Contents Available at: http//www.bbrc.in/

Doi: $h t t p: / / d x$.doi.org/10.21786/bbrc/13.8/132
2012). These technologies allow the clinicians to provide computer-driven customized care solutions at varying levels (Sachdeva et al., 2012). Suresmile technology is one of the latest advanced technologies in orthodontic treatment which has been introduced since 1998 and commercially released in 2005 (Alford et al., 2011). It was introduced by Dr. Sachdeva who is the co-founder and Chief Clinical Officer of Suresmile (Scholz and Sachdeva, 2010). In most of his publications, he stated that Suresmile technology is designed to minimize the errors during orthodontic treatment due to appliance management as well as shorten the time of the treatment.

Besides, it is also believed to provide a completely integrated, clinical solution to the extended care cycle (Sachdeva et al., 2012). Based on the study done, although

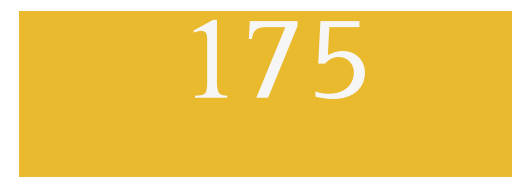


Suresmile patients may have a shorter treatment time, the quality is still maintained at its best. In an interview done between Dr. Sachdeva and Robert P, he explained that the reason behind Suresmile technology's invention is to speed up an extremely careful delivery model (Scholz and Sachdeva, 2010). Through the Suresmile system, orthodontic archwire-assisted forming equipment and orthodontic archwire bending machines have been used to bend orthodontic archwires (Jiang et al., 2019). The robot is activated and worked according to the input inserted by the dentist. Therefore, it leads to careful diagnosis and a well-designed treatment plan.

In orthodontics, three dimensional (3D) digital models are widely used for the purpose of storage, diagnosis, design of custom appliances and orthodontic treatment outcome evaluations (Scholz, 2009; Scholz and Sachdeva, 2010; Alford et al., 2011; Grauer and Proffit, 2011). The uses of various types of scanner such as structured or unstructured laser scanners, tabletop scanners, optical scanners and industrial-grade CAT scanner helps in capturing the digital representation of the 3D model (Sachdeva et al., 2012; Hayashi et al., 2013). In Suresmile technology, Suresmile OraScanner (OraMetrix, Richardson, Tex) is used to create a threedimensional representation of the dentition. It is an optical scanner with a structured white light that can be used to scan the dentition and physical model in vivo and in vitro (Hayashi et al., 2013). Apart from that, various company has introduced another optical scanner in the marketplace with a similar function such as iTero (Align Technology, San Jose, Calif), CEREC (Sirona, Bensheim, Germany) and Lava COS (3M Unitek, St Paul, Minn) (Garg, 2008; van der Meer et al., 2012). Meanwhile, the tabletop scanner created a three-dimensional (3D) model by capturing the three dimensional (3D) images of the impressions or physical models. The advantage of this scanner is it has the ability to minimize the blind area through the auto-rotating unit (Hayashi et al., 2013).

This cutting-edge technology is a three-dimensional visualization that helps in the diagnosis of orthodontic patients, image capturing, monitoring and patient communication and also provides appliances with precision (Sachdeva, 2001). Along with OraScanner and OraScan Image Processing software, it gives a complete vision of the treatment. Apart from Suremile OraScanner, a Cone Beam Computed Tomography (CBCT) can also be used in vivo or in vitro to create a 3D model of the patients' dentition and appliances on each individual tooth (Hayashi et al., 2013). It is important to understand the accuracy and reliability of these imaging devices. These scanners might affect the accuracy and precision of the customized appliance in which the three-dimensional models of the appliance are designed based on the image captured. Thus, proper knowledge about this technology is at utmost essential to ensure the great and successful outcome of the orthodontic treatment (Hayashi et al., 2013).

Suresmile technology uses a customized archwire and a usual conventional bracket on the patient with orthodontic problems. The customized arch-wire is made up of a wire bending robot based on the orthodontist's preferences. This robotic technology is very helpful to the orthodontist in which the limitations of precision, accuracy and reliability when performing a manual and repetitive tasks at the chairside can be overcome (Scholz and Sachdeva, 2010). For example, archwire bending requires a long period of time manually. The process of Suresmile begins after the patient's dentition is scanned using OraScanner. The scan data are then used to locate the bracket on each individual tooth and construct a digital model of the patient's dentition, known as the therapeutic model (Larson, Vaubel and Grünheid, 2013). It allows for detailed treatment planning and the operator allows the tooth to move independently in three dimensions (Mah and Sachdeva, 2001). Multiple treatment scenarios can be designed by the operator based on his diagnosis and knowledge. The operator can also simulate different treatment plans until the optimal one is found (Mah and Sachdeva, 2001).

Apart from various latest technologies, there is also a lot of research that has been done that focused on the movement of teeth in orthodontic treatment to reduce the treatment time. The possible interventions for accelerating tooth movement can be classified into a surgical and no-surgical method (Gkantidis et al., 2014). The mechanisms in the movement of teeth include the physics of force application and the biological response of the dentoalveolar tissues to this force (Miles, 2017). Examples for a non-surgical method are limited orthodontic treatment, self-ligating and various bracket design, customized appliance, medication, micro-vibration, low-intensity laser, photobiomodulation (PBM), electromagnetic fields and direct electric currents (Miles, 2017).

Meanwhile, the surgical interventions are corticotomy, distraction osteogenesis, piezocision, PDL distraction and micro-osteoperforations (Miles, 2017). Recently, nanorobot might also involve in the acceleration of tooth movement which is currently under research due to the demand for shorter orthodontic treatment. Currently, patients with fixed appliance treatment, the mean duration of treatment would be 2-3 years exact or more if some circumstances occur (Dhayananth and Chajallani, 2016). Thus, it will result in various problems such as scars of orthodontic treatment, gingival inflammation and root resorption (Dhayananth and Chajallani, 2016). In orthodontics, nanorobots help in reducing the orthodontic treatment time. The periodontal tissue is directly manipulated in which it facilitates the movement and painless tooth straightening, rotating and vertical repositioning in just hours or minutes (Dhayananth and Chajallani, 2016).

The introduction of digital models into dentistry which is capable of capturing both radiographic and facial images is on the horizon. Besides, the uses of integrated software to review these different images and intraoral scans make huge changes in this industry. This kind of software is very useful in the analysis of patient, treatment 
planning for orthodontic, prosthetic and surgical patient and designing and fabricating the dental restorations, prosthetic devices, dentures, temporomandibular joint (TMJ) splint, implant placement splints, surgical splints and fabrication of orthodontic appliances (Hero Breuning and Kau, 2017). The use of Suresmile (OraMetrix, Inc, Richardson, TX) or three dimensional (3D) system in orthognathic surgery has gained attention in the industry (Maron, Kuhmichel and Schreiber, 2017). Through this, the patients are able to know the possible results of the treatment before starting with the treatment.

Our extensive research expertise ranged from epidemiological studies to randomized clinical trials that have been published in reputed journals (Felicita, 2017a, 2017b, 2018; Felicita, Thirumurthi and Jain, 2017; Korath, Padmanabhan and Parameswaran, 2017; Krishnan, Pandian and Rajagopal, 2017; Charles et al., 2018; Pandian, Krishnan and Kumar, 2018; Reddy et al., 2018; Chinnasamy et al., 2019). This knowledge was instrumental for us to study the effectiveness and the efficiency between the conventional method and Suresmile technology.

Suresmile Technology

Development of Suresmile technology: The idea of Suresmile germinated from a meeting of forwardthinking experts on orthodontic and technology. They also visualized a system through customizing shape memory alloy wires. It is believed to help in improving the quality and predictability of orthodontic treatment (Claudia \oaita, 2012). It was introduced by Dr. Sachdeva, an orthodontist and Chief Officer of the Suresmile. He came up with the idea after returning from a lecture tour in India by Professor Reggie Miethke from the Free University of Berlin. After the exposure of knowledge about the first generation of robotic archwire bending technology, he believed that there is a high possibility to develop a new model of orthodontic care delivery through mass customization. In the late 1990s, he began with only two people in Richardson, Texas and later he joined forces with a German company named OraMetrix led by Chuck Abraham (Scholz and Sachdeva, 2010). After Suresmile technology has been commercialized in near 2005, most of the orthodontic patients are willing to pay greater cost due to the shorter treatment times. Now, it has been introduced in different countries such as the United States, Canada, Australia and Germany (Scholz and Sachdeva, 2010).

OraScan Image Processing Software will show an accurate, three-dimensional representation of the dentition (Sachdeva, 2001). There are many functional components in this treatment planning software such as three-dimensional visualization, measurement, communication, decision making with simulation, bracket placement, setup and archwire design, quality and outcome assessment and Suresmile patient management (Scholz and Sachdeva, 2010). These components can be used either in a single or in a combination of various components which allows the dentist to come out with better decisions and design the targeted prescription archwire (Scholz and Sachdeva, 2010). In three-dimensional visualization utility, it allows the dentist to view the multiple projections of the arches and dentition. The advantage of this utility is it allows a simultaneous assessment of the spatial position of the tooth and its morphology accurately on how it affects the occlusal scheme of each different patient (Scholz and Sachdeva, 2010).

Besides, the tooth widths are measured along with Bolton analysis automatically. Therefore, the orthodontists can achieve a better idea and visualization of the various treatment plans for their patients which can be easily done in less than ten minutes using all these setup components. One interesting outcome of this software is the communication between orthodontists and patients. Through this, the patient could understand their own problems and thus, making them active throughout the journey with the orthodontist. The patients can make their own decision with the knowledge exposed to them through visualization and interactive simulation tools.

The two approaches in using Suresmile technology in the clinical practice include i) comprehensive system and ii) indirect bonding tray with the aligning and finishing wires (Nguyen and Jackson, 2018). During the first appointment with an orthodontist, a quick scan of dentition using OraScanner, a handheld device is taken. It is able to create an exact replica of the dentition and is relatively independent which means the picture produced is not affected by any movement, if presence (Sachdeva, 2001). Then, by using the OraScan Image Processing Software, the images were combined to produce a threedimensional image. Through this, the clinician is able to plan for the treatment based on the parameters such as midline, occlusal plane level and arch dimension (Mah and Sachdeva, 2001). A three-dimensional digital diagnostic setup is created as the final treatment plan before proceeding with bonding.

The automatic digital bonding feature is activated after satisfactory target positions. All the data including the location and retention required by the brackets and wire are sustained in the computer and sent to the Suresmile office (Abutayyem et al., 2019). Then, the arch-wire geometry is automatically calculated in three dimensions for the bracket positions on the target arch (Sachdeva, 2001). The position of each tooth is further refined in the final phase of digital treatment planning. There may be changes in the bracket position or arch-wire geometry. Prescription wires and a blank tray is used for indirect bonding. The brackets are filled in the tray and bonded using conventional indirect bonding as it is an accurate and fast method compared to others. The orthodontist may use OraScanner during follow up appointments to observe the progress and correct the errors if any. During the finishing stage, custom wires are formed by a wire bending robot that will individualize the treatment to achieve the desired outcome (Scholz and Sachdeva, 2010). 
The wire bending robot is known as the Suresmile archwire bending robot. It can automatically change into a particular shape according to the preferences. The gripping tools can be divided into two types. In the combination of force sensors, the gripping tool helps to determine over bends in order to achieve the desired shape of the archwire. In addition, the presence of a resistive heating system allows maintaining the bent shape of the archwire through heat which is supplied by the current (Jiang et al., 2015). Suresmile (OraMetrix) is one of the customized orthodontic technologies which help the orthodontist to achieve an ideal alignment, aesthetics and occlusion for their patients.

Advantages of Suresmile Technology: This technology is most preferred by orthodontists and patients because the advantages outweigh the disadvantages. Grünheid, Thorsten, et al. stated that Suresmile provides the best combination of accuracy, reproducibility, and time efficiency of measurement (Grünheid et al., 2014). The accuracy was measured by using the Bland-Altman method (Bland and Altman, 1999). Mean squared error was calculated to rank the measurement of the accuracy: mean squared error = bias 2 + variance. Suresmile has the highest reproducibility in comparison with other digital models such as emodel and AnatoModel as it has the smallest mean squared error. The efficiency of the Suresmile is ranked second as it takes a longer time to complete the tooth size measurement compared to emodel.

In a study done by Kazua Hayashi et al, they had assessed the accuracy and reliability of OraScanner (OraMetrix, Inc. Richardson, TX) by comparing it with other three dimensional scanners such as VIVID910 (Konica Minolta, Tokyo, Japan) and R700 (3Shape, Copenhagen, Denmark) (Hayashi et al., 2013). The gold standard scanner, SLP250 Laser Probe (Laser Design, Detroid, Mich) was also compared. The dental casts were used in this study as it plays an important role in diagnosis and also treatment outcomes. The results showed that there were slight differences in mean value with $<0.057 \mathrm{~mm}$ in comparison to the gold standard scanner. Thus, this study suggested that OraScanner VIVID910 and R700 were accurate and can be used by the orthodontist without problems (Hayashi et al., 2013).

Orthodontists believe that the main advantage of Suresmile technology is that they have choices to use their preferred bracket system, as well as it may increase precision in the finishing stage (Alford et al., 2011). Besides, the customized wire can be created by getting information through an intra-oral scan or cone-beam computed tomography (CBCT) (Grauer and Proffit, 2011). It can be designed to be active in the anterior segment with the desired alignment and passive in the posterior segment with the ideal occlusion of the patient.

Disadvantages of Suresmile Technology: The disadvantages of Suresmile Technology includes the possibility of debonding of the bracket after archwire customization and before completion of treatment (Grauer and Proffit, 2011).

Effectiveness of Suresmile Technology to achieve predicted outcomes: The predicted outcomes of the tooth position in orthodontic patients are varied according to tooth type and dimension of movement. In Suresmile, the vertical dimension is the most effective tooth movement. Some outcomes treated with conventional fixed appliances show some deficiency in the vertical dimension. The actual treatment outcome for canine and premolars, it turned out to be positioned apically from the virtual treatment plan. In cases of incisors and molars, the actual treatment outcome tends to be positioned occlusally. In the buccolingual dimension, all teeth except for maxillary incisor and mandibular second molars; it turned out to be positioned lingually in the actual treatment plan (Larson, Vaubel and Grünheid, 2013).

Suresmile is a computer-aided appliance that helps to produce precision in arch-wire bending. However, it does not prevent the needs of auxiliary mechanics. Interarch elastic is one of the Class II mechanics and commonly used in Suresmile technology (Larson, Vaubel and Grünheid, 2013). Suresmile technology is able to solve problems if there are errors in bracket placement and variations in adhesive thickness (Mah and Sachdeva, 2001). Meanwhile, treatment planning, appropriate diagnosis depends on the capability of the orthodontist.

Suresmile vs Conventional Method-Treatment time: Length of treatment time is one of the factors which may be considered by the orthodontic patients (Skidmore et al., 2006). It is believed that shorter treatment time will help in efficiency, effectiveness and timeliness quality care (Baker, 2001). However, extended length of care may give disadvantages to the patients as well as poor quality care (Fox, 2005)). Clearly, in conventional methods, the repositioning of the brackets, altering bracket prescriptions or archwire bending contribute to the successful treatment outcomes. However, in the Suresmile method, the customized archwire is made using the input setup and prescribed to the patient (Sachdeva et al., 2012). This will lead to an earlier control of the tooth movement. Besides, with several practices, the orthodontist may also reduce the treatment time.

Sachdeva et al recently reported the comparison of treatment time between Suresmile and Conventional bracket systems. The author analyzed a total of 12,335 patients which 9,390 patients underwent Suresmile treatment and 2,945 patients with conventional treatment. All the data of the patients were recorded including treatment time, malocclusion class, patient age and patient visits. The patients with complete data were chosen for further analysis. The results approved that Suresmile technology had a greater efficiency as the treatment time in Suresmile patients were 15 months of median treatment time and conventional patients with 23 months of median treatment time with 
a significant difference of 8 months median treatment time. Besides, in malocclusion class by Angle showed that there was evidence of shorter treatment time in all three classes with the mean difference of 8 and 9. For class I malocclusion, Suresmile showed a mean value of 16 months and conventional shows a mean value of 24 months.

In class II malocclusion, Suresmile showed a mean value of 14 months and conventional shows a mean value of 22 months. Lastly, in class III malocclusion, the Suresmile showed a mean value of 17 months and conventional showed a mean value of 25 months. Class II patients had a shorter median time compared to class I and class III. This may be due to only mild class II patients being involved compared to class I which may have more severe crowding. Based on age, the previous study revealed that both adolescents and adults showed a significant difference in the treatment time between Suresmile and conventional with a mean difference of 8 and 9 respectively. The mean value of Suresmile in adolescents and adults shows a mean of 16 and 17 months respectively. Besides, this study also shows that the treatment visits of the patient using Suresmile can be reduced by a median of eight months in comparison to the conventional method. Suresmile patients required only 14 visits throughout the treatment and conventional required at least 18 visits throughout treatment. Thus, this study provided evidence that the variables including malocclusion class and age in patient visits in Suresmile patients were shorter compared to conventional patients (Sachdeva et al., 2012).

In addition to that, the other treatment modalities in orthodontic patients include the requirement of extraction or any surgical procedure. A study done by Fink and Smith suggested additional months of treatment time were required. The extraction of single premolar, two premolars and four premolars results in an additional of 0.9, 1.8 and 3.6 months respectively. However, the treatment time did not exceed the conventionally treated patients (Fink and Smith, 1992).

Besides, Saxe et al recently analyzed a total of 62 patients, Suresmile [38] and conventional [24] treated patients based on pre and post-treatment study. Discrepancy index (DI) and Objective Grading System (OGS) was determined by the treatment study respectively. According to the ABO OGS scores, Suresmile showed a mean of 26.3 with a significant difference of 4.4 from conventional treatment with 30.7. Suresmile patients demonstrated an OGS score of $14.3 \%$ better than the conventional method. This suggests that better outcomes are not related to long treatment time. Meanwhile, the treatment time showed a difference of almost six months, Suresmile had a treatment time of 14.7 months with a standard deviation of 4.71 months and conventionally had a treatment time of 20.0 months with a standard deviation of 6.40 months. This suggests a decrease in mean treatment time by $36 \%$. The level of difficulty which was measured using DI does not influence the treatment result (Saxe, Louie and Mah, 2010).
In addition, 132 non-extraction patients were studied by Alford et al which were divided into Conventional and Suresmile methods. 63 patients were treated using Conventional, meanwhile Suresmile composed 69 patients. This study showed that Suresmile has a shorter treatment time which is 15.8 months compared to 23 months in the conventional method. Cast/radiographic evaluation (CRE) is suggested as one of the effective implementations to evaluate different clinical outcomes. Since male had a higher percentage in conventional (51\%) compared to Suresmile (38\%), thus based on this study, male patients in the conventional method were likely to have higher CRE scores. The reasons behind this are due to the exposure towards complex occlusion and larger fractions (Alford et al., 2011).

In some circumstances, there might be an increase in treatment time due to the problem of the inaccuracy of bending arch-wire. This may result in an iatrogenic problem for the patients. Suresmile robotic devices tend to preserve superelastic properties in arch-wires which provide the best alignment of the crowns of the teeth compared to manual bent arch-wires. The conventional method is more efficient than Suresmile in terms of root angulation (Alford et al., 2011). In Suresmile, the crown is effectively tipped to the desired position, but the root is not well aligned due to some problems.

\section{CONCLUSION}

In conclusion, advancement in orthodontics such as Suresmile technology enables the orthodontist and patients to have an effective and efficient treatment outcome. The Ora Scanner by Suresmile also provides the utmost accuracy in comparison to the gold standard which allows the clinician to implement Suresmile without any doubt. It has been used widely by orthodontists and it may be expanded all over the world in the future. The advantages of this technology which provides benefits to the patients especially in terms of length of treatment make the patient willing to pay greater cost just for shorter treatment time. They might feel comfortable throughout the treatment as the number of visits to the dentist can also be reduced by using Suresmile. In the future, improvisation in Suresmile technology such as man-machine interface, more intelligent design of the software and unified interface for the choice of customized appliances could be done (Scholz and Sachdeva, 2010). Therefore, further research on Suresmile technology can be done in order to evaluate the effectiveness, reliability, accuracy and efficiency of this technology.

\section{ACKNOWLEDGEMENTS}

We thank Saveetha Dental College and Hospitals, Saveetha Institute of Medical and Technical Sciences, Saveetha University for granting the research proposal and research support.

Conflict of Interest: No conflict of interest declared by the authors. 


\section{REFERENCES}

Abutayyem, H. et al. (2019) 'Robotic Use in Orthodontics: Literature Review’, Oral Health Dental Sci. researchgate. net, 3(2), pp. 1-5.

Alford, T. J. et al. (2011) 'Clinical outcomes for patients finished with the SureSmileTM method compared with conventional fixed orthodontic therapy', The Angle orthodontist. Allen Press, 81(3), pp. 383-388.

Baker, A. (2001) 'Crossing the Quality Chasm: A New Health System for the 21st Century', BMJ . British Medical Journal Publishing Group, 323(7322), p. 1192.

Bland, J. M. and Altman, D. G. (1999) 'Measuring agreement in method comparison studies', Statistical methods in medical research. journals.sagepub.com, 8(2), pp. 135-160.

Charles, A. et al. (2018) 'Evaluation of dermatoglyphic patterns using digital scanner technique in skeletal malocclusion: A descriptive study', Indian journal of dental research: official publication of Indian Society for Dental Research, 29(6), pp. 711-715.

Chinnasamy, A. et al. (2019) 'Chronic nail biting, orthodontic treatment and Enterobacteriaceae in the oral cavity', Journal of clinical and experimental dentistry, 11(12), pp. e1157-e1162.

Claudia \OAITA, S. P. (2012) 'Some aspects of computer aided engineering techniques in orthodontic treatment', in The 6th edition on the Interdisciplinarity in Engineering International Conference, pp. 412-415.

Dhayananth, L. X. and Chajallani, V. G. (2016) 'Renaissance in orthodontics: Nanotechnology', Aquatic microbial ecology: international journal. orthodrehab. org..

Felicita, A. S. (2017a) 'Orthodontic management of a dilacerated central incisor and partially impacted canine with unilateral extraction - A case report', The Saudi dental journal, 29(4), pp. 185-193.

Felicita, A. S. (2017b) 'Quantification of intrusive/ retraction force and moment generated during enmasse retraction of maxillary anterior teeth using mini-implants: A conceptual approach', Dental press journal of orthodontics, 22(5), pp. 47-55.

Felicita, A. S. (2018) 'Orthodontic extrusion of Ellis Class VIII fracture of maxillary lateral incisor - The sling shot method', The Saudi dental journal, 30(3), pp. 265-269.

Felicita, A. S., Thirumurthi, A. S. and Jain, R. K. (2017) 'Patient's Psychological Response to Twinblock Therapy', World Journal of Dentistry, 8(4), pp. 327-330.

Fink, D. F. and Smith, R. J. (1992) 'The duration of orthodontic treatment', American journal of orthodontics and dentofacial orthopedics: official publication of the American Association of Orthodontists, its constituent societies, and the American Board of Orthodontics. ajodo.org, 102(1), pp. 45-51.

Fox, N. (2005) 'Longer orthodontic treatment may result in greater external apical root resorption', Evidencebased dentistry. nature.com, p. 21.

Garg, A. K. (2008) 'Cadent iTero's digital system for dental impressions: the end of trays and putty?', Dental implantology update. europepmc.org, 19(1), pp. 1-4. Gkantidis, N. et al. (2014) 'Effectiveness of nonconventional methods for accelerated orthodontic tooth movement: a systematic review and meta-analysis', Journal of dentistry. Elsevier, 42(10), pp. 1300-1319.

Grauer, D. and Proffit, W. R. (2011) 'Accuracy in tooth positioning with a fully customized lingual orthodontic appliance', American journal of orthodontics and dentofacial orthopedics: official publication of the American Association of Orthodontists, its constituent societies, and the American Board of Orthodontics. Elsevier, 140(3), pp. 433-443.

Grünheid, T. et al. (2014) 'Accuracy, reproducibility, and time efficiency of dental measurements using different technologies', American journal of orthodontics and dentofacial orthopedics: official publication of the American Association of Orthodontists, its constituent societies, and the American Board of Orthodontics. Elsevier, 145(2), pp. 157-164.

Hayashi, K. et al. (2013) 'Assessment of the accuracy and reliability of new 3-dimensional scanning devices', American journal of. Elsevier.

Hero Breuning, K. and Kau, C. H. (2017) Digital Planning and Custom Orthodontic Treatment. John Wiley \& Sons.

Jiang, J. et al. (2019) 'Digital expression and interactive adjustment method of personalized orthodontic archwire for robotic bending', Journal of Advanced Mechanical Design, Systems, and Manufacturing. jstage.jst.go.jp, 13(2), pp. JAMDSM0031-JAMDSM0031.

Jiang, J.-G. et al. (2015) 'A Review on Robot in Prosthodontics and Orthodontics', Advances in Mechanical Engineering. SAGE Publications, 7(1), p. 198748.

Korath, A. V., Padmanabhan, R. and Parameswaran, A. (2017) 'The Cortical Boundary Line as a Guide for Incisor Re-positioning with Anterior Segmental Osteotomies', Journal of maxillofacial and oral surgery, 16(2), pp. 248-252.

Krishnan, S., Pandian, S. and Rajagopal, R. (2017) 'Sixmonth bracket failure rate with a flowable composite: A split-mouth randomized controlled trial', Dental press journal of orthodontics, 22(2), pp. 69-76.

Larson, B. E., Vaubel, C. J. and Grünheid, T. (2013) 'Effectiveness of computer-assisted orthodontic treatment technology to achieve predicted outcomes', The Angle orthodontist. meridian.allenpress.com, 83(4), pp. 557-562.

Mah, J. and Sachdeva, R. (2001) 'Computer-assisted orthodontic treatment: the SureSmile process', American journal of orthodontics and dentofacial orthopedics: official publication of the American Association of Orthodontists, its constituent societies, and the 
American Board of Orthodontics. Elsevier, 120(1), pp. 85-87.

Maron, G., Kuhmichel, A. and Schreiber, G. (2017) 'Secondary Treatment of Malocclusion/Malunion Secondary to Condylar Fractures', Atlas of the oral and maxillofacial surgery clinics of North America. jawsoms. com, 25(1), pp. 47-54.

van der Meer, W. J. et al. (2012) 'Application of intra-oral dental scanners in the digital workflow of implantology', PloS one. journals.plos.org, 7(8), p. e43312.

Miles, P. (2017) 'Accelerated orthodontic treatment-what's the evidence?', Australian dental journal. Wiley Online Library.

Nguyen, T. and Jackson, T. (2018) '3D technologies for precision in orthodontics', Seminars in orthodontics. Elsevier, 24(4), pp. 386-392.

Pandian, K. S., Krishnan, S. and Kumar, S. A. (2018) 'Angular photogrammetric analysis of the soft-tissue facial profile of Indian adults', Indian journal of dental research: official publication of Indian Society for Dental Research, 29(2), pp. 137-143.

Reddy, A. K. et al. (2018) 'Comparative Evaluation of Antimicrobial Efficacy of Silver, Titanium Dioxide and Zinc Oxide Nanoparticles against Streptococcus mutans', Pesquisa brasileira em odontopediatria e clinica integrada, 18(1), p. e4150.

Sachdeva, R. C. (2001) 'SureSmile technology in a patient--centered orthodontic practice', Journal of clinical orthodontics: JCO. jco-online.com, 35(4), pp. 245-253.

Sachdeva, R. C. L. et al. (2012) 'Treatment time: SureSmile vs conventional', Orthodontics-the Art and Practice of Dentofacial Enhancement. researchgate.net, 13, p. 72.

Saxe, A. K., Louie, L. J. and Mah, J. (2010) 'Efficiency and effectiveness of SureSmile', World journal of orthodontics. jgajera.angelfire.com, 11(1), pp. 16-22.

Scholz, R. P. (2009) 'Interview with a SureSmile doctor: Nicole M. Jane', American journal of orthodontics and dentofacial orthopedics: official publication of the American Association of Orthodontists, its constituent societies, and the American Board of Orthodontics. Elsevier, 135(4, Supplement), pp. S140-S143.

Scholz, R. P. and Sachdeva, R. C. L. (2010) 'Interview with an innovator: SureSmile chief clinical officer Rohit CL Sachdeva', American journal of orthodontics. ajodo. org.

Skidmore, K. J. et al. (2006) 'Factors influencing treatment time in orthodontic patients', American journal of orthodontics and dentofacial orthopedics: official publication of the American Association of Orthodontists, its constituent societies, and the American Board of Orthodontics. Elsevier, 129(2), pp. 230-238. 